Supporting information

\title{
Photoactive and antioxidant nanochitosan dots/biocellulose hydrogels for wound healing treatment
}

Danica Z. Zmejkoski ${ }^{*}$, Zoran M. Markovića ${ }^{\mathrm{a}}$, Milica D. Budimir ${ }^{\mathrm{a}}$, Nemanja M. Zdravković ${ }^{\mathrm{b}}$, Dijana D. Trišićc ${ }^{c}$ Nikol Bugárovád, Martin Danko ${ }^{d}$, Natalia O. Kozyrovska ${ }^{\mathrm{e}}, Z_{\text {Zdeno Špitalskýd }}^{\mathrm{d}}$, Angela Kleinovád, Sanja B. Kuzman ${ }^{\mathrm{a}}$, Vladimir B. Pavlović ${ }^{\mathrm{f}}$, Biljana M. Todorović Marković ${ }^{\mathrm{a}^{*}}$ ${ }^{\mathrm{a}}$ Vinča Institute of Nuclear Sciences - National Institute of the Republic of Serbia, University of Belgrade, P.O.B. 522, 11001 Belgrade, Serbia; danica@vin.bg.ac.rs, zoranmarkovic@ vin.bg.ac.rs, mickbudimir@gmail.com, sanjaculubrk@gmail.com, biljatod@vin.bg.ac.rs

${ }^{\mathrm{b}}$ Scientific Veterinary Institute of Serbia, Janisa Janulisa 14, 11107 Belgrade, Serbia; nemanja.zdravkovich@gmail.com

${ }^{c}$ Faculty of Dental Medicine, University of Belgrade, Dr. Subotića 8, 11000 Belgrade, Serbia; dijana.trisic@stomf.bg.ac.rs

${ }^{\text {d}}$ Polymer Institute, Slovak Academy of Sciences, Dúbravska cestá 9, 84541 Bratislava, Slovakia; nikola.bugarova@savba.sk, upoldan@savba.sk, zdeno.spitalsky@savba.sk, upolklan@savba.sk

${ }^{\mathrm{e}}$ Institute of Molecular Biology and Genetics, National Academy of Sciences of Ukraine, 150, Zabolotnogo Str., Kyiv, Ukraine, 03143; kozyrna@ukr.net

${ }^{\mathrm{f}}$ Faculty of Agriculture, University of Belgrade, Nemanjina 6, 11080 Belgrade-Zemun, Serbia; vlaver@agrif.bg.ac.rs

\footnotetext{
* Corresponding authors : biljatod@vin.bg.ac.rs (B. Todorović Marković); danica@vin.bg.ac.rs (D. Zmejkoski)
} 

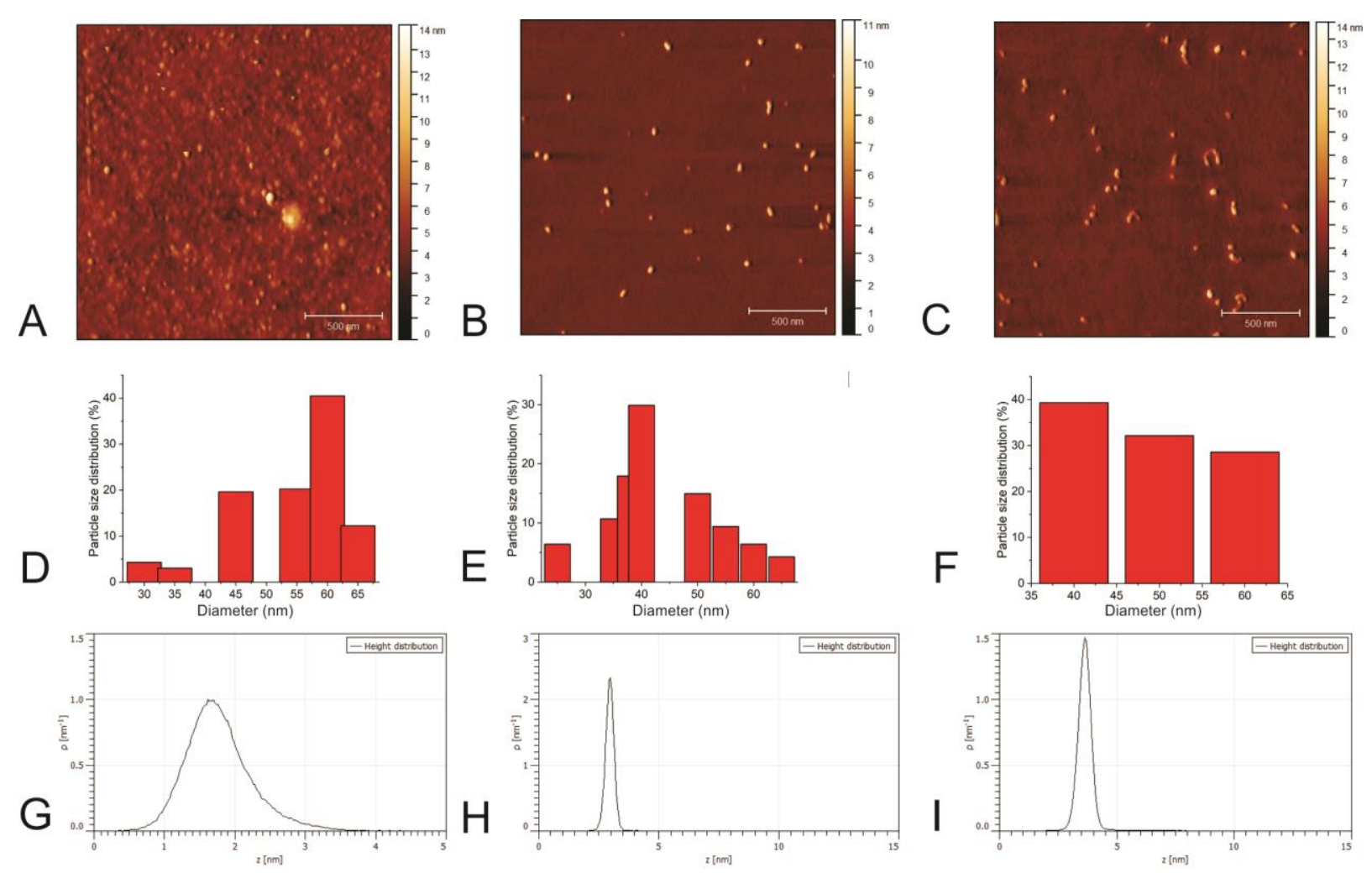

Fig. S1. Top view AFM images of n20ChiD (A), n40ChiD (B) and n60ChiD (C) samples; particle size distribution of n20ChiD (D), n40ChiD (E) and n60ChiD (F) samples; height profiles of n20ChiD (G), n40ChiD (H) and n60ChiD (I) samples. 


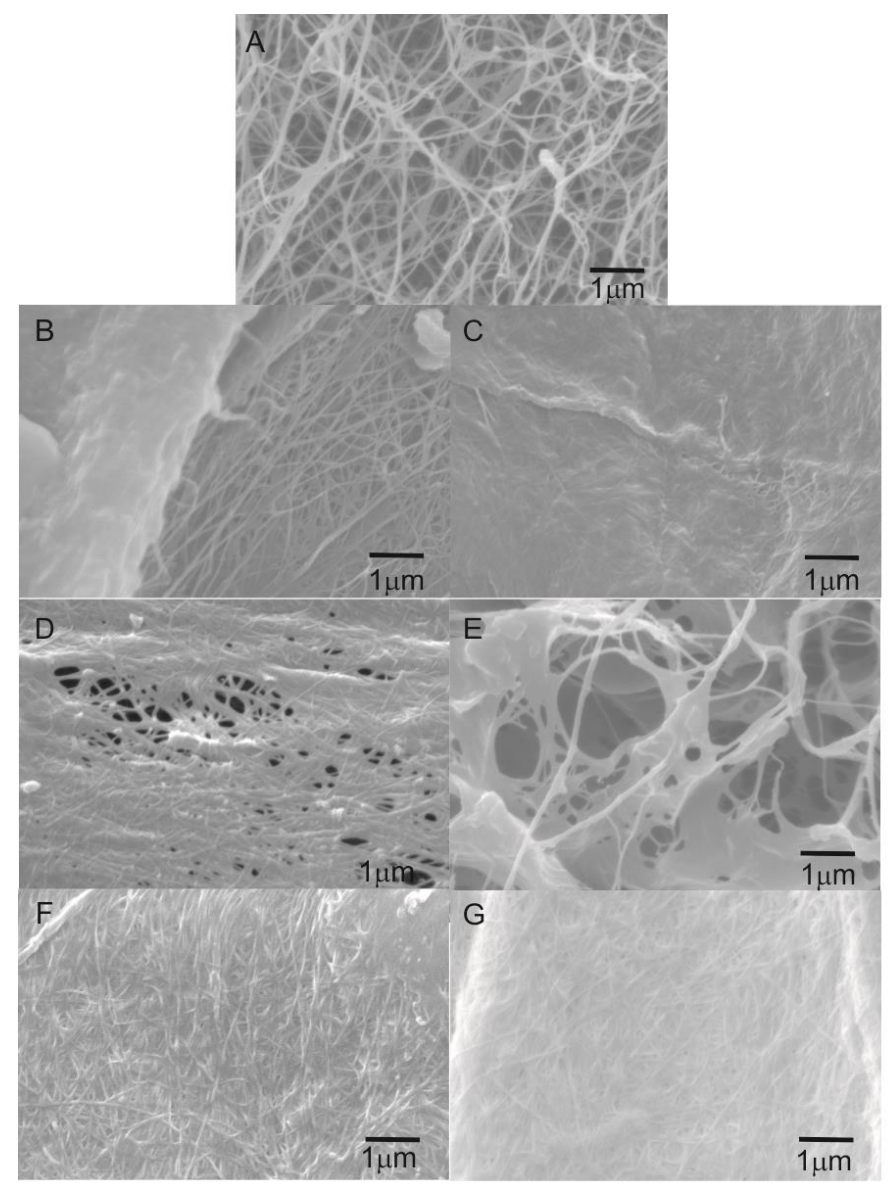

Fig. S2. SEM micrographs of A) BC, B) BC-n20ChiD0.2, C) BC-n20ChiD2, D) BCn40ChiD0.2, E) BC-n40ChiD2, F) BC-n60ChiD0.2 and G) BC-n60ChiD2 samples. 
A

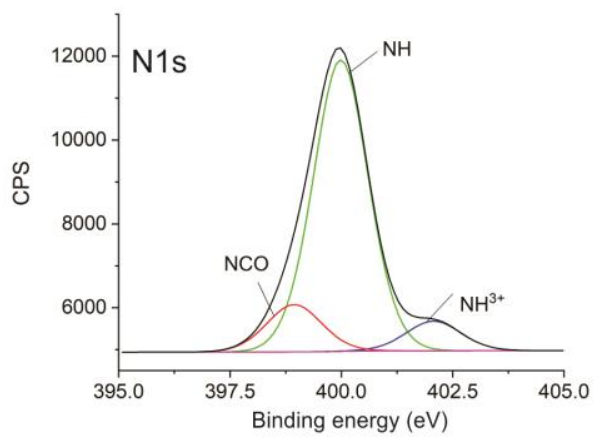

C

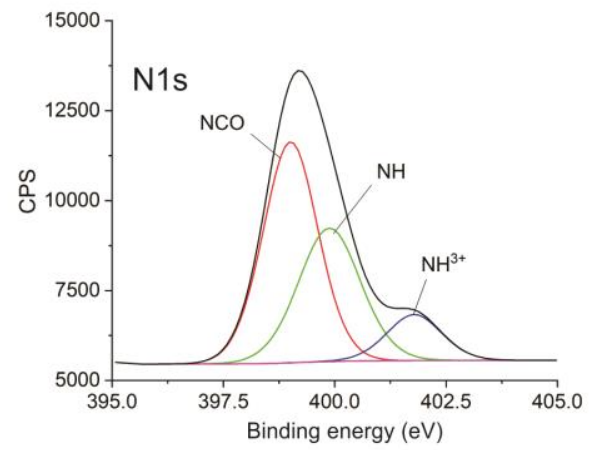

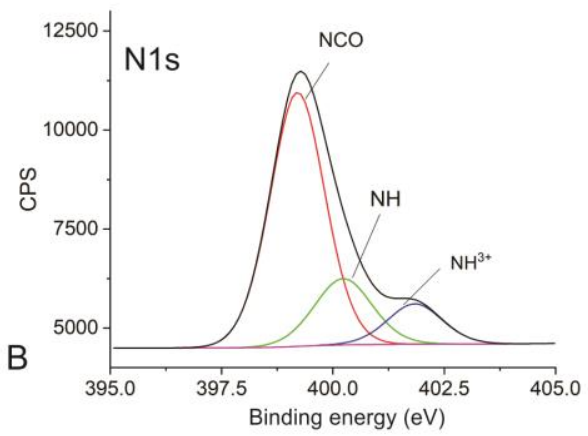

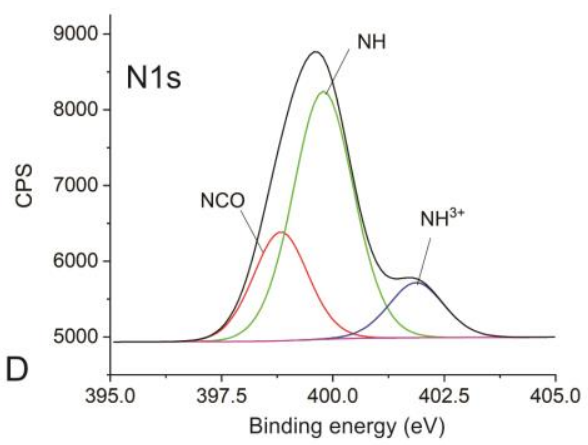

Fig. S3.The high-resolution XPS spectra of N1s peak of (A) BC, (B) BC-n20ChiD2, (C) BCn40ChiD2 and (D) BC-n60ChiD2.

Table S1. Elements detected in BC, BC-n20ChiD2, BC-n40ChiD2 and BC-n60ChiD2 samples by XPS method.

\begin{tabular}{|l|l|l|l|l|l|l|l|}
\hline Name BC & Atomic\% & $\begin{array}{l}\text { Name BC- } \\
\text { 20nChiD2 }\end{array}$ & Atomic\% & $\begin{array}{l}\text { Name BC- } \\
\text { 40nChiD2 }\end{array}$ & Atomic\% & $\begin{array}{l}\text { Name BC- } \\
\text { 60nChiD2 }\end{array}$ & Atomic\% \\
\hline C1s & 59.5 & C1s & 73.4 & C1s & 73.3 & C1s & 67.4 \\
\hline O1s & 36.8 & O1s & 21.8 & O1s & 21.5 & O1s & 30.7 \\
\hline N1s & 3.7 & N1s & 4.8 & N1s & 5.2 & N1s & 2.5 \\
\hline
\end{tabular}

Table S2. Characteristic bonds detected in C1s and N1s peaks of BC sample by XPS method.

\begin{tabular}{|l|r|r|}
\hline Name BC & $\begin{array}{l}\text { Binding } \\
\text { energy } \\
(\mathrm{eV})\end{array}$ & $\begin{array}{l}\text { Atomic } \\
(\%)\end{array}$ \\
\hline $\mathrm{C} 1 \mathrm{~s}$ C-C & 284.8 & 7.3 \\
\hline $\mathrm{C} 1 \mathrm{~s}$ C-O, C-N & 286.4 & 24.5 \\
\hline $\mathrm{C} 1 \mathrm{~s}$ O-C-O, NCO & 287.9 & 6.5 \\
\hline $\mathrm{C} 1 \mathrm{~s}$ O-C=O & 289.0 & 0.9 \\
\hline O1s C-O & 532.7 & 52.4 \\
\hline
\end{tabular}




\begin{tabular}{|l|r|r|}
\hline O1s -CO- & 531.2 & 2.2 \\
\hline O1s O2*CO & 533.5 & 4.2 \\
\hline $\mathrm{N} 1 \mathrm{~s} \mathrm{NH}$ & 400.0 & 2.1 \\
\hline $\mathrm{N} 1 \mathrm{~s} \mathrm{NCO}$ & 398.9 & 0.4 \\
\hline $\mathrm{N}^{\mathrm{s}} \mathrm{NH}^{3+}$ & 402.1 & 0.2 \\
\hline
\end{tabular}

Table S3. Characteristic bonds detected in C1s and N1s peaks of BC-n20ChiD2 sample by XPS method.

\begin{tabular}{|l|l|l|}
\hline Name BC-n20ChiD2 & Binding energy $(\mathrm{eV})$ & Atomic $(\%)$ \\
\hline C1s C-C & 284.5 & 28.9 \\
\hline C1s C-O, C-N & 286.2 & 20.8 \\
\hline C1s O-C-O, NCO & 287.9 & 5.2 \\
\hline C1s O-C=O & 289.0 & 0.6 \\
\hline O1s C-O & 532.5 & 36.2 \\
\hline O1s -CO- & 531.0 & 2.9 \\
\hline O1s O2*CO & 533.4 & 2.1 \\
\hline N1s NCO & 399.2 & 2.9 \\
\hline N1s NH & 400.2 & 0.8 \\
\hline N1s NH3+ & 401.9 & 0.5 \\
\hline
\end{tabular}

Table S4. Characteristic bonds detected in C1s and N1s peaks of BC-n40ChiD2 sample by XPS method.

\begin{tabular}{|l|l|l|}
\hline Name BC-n40ChiD2 & Binding energy (eV) & Atomic (\%) \\
\hline C1s C-C & 284.4 & 29.8 \\
\hline C1s C-O, C-N & 286.1 & 21.3 \\
\hline C1s O-C-O, NCO & 287.8 & 5.4 \\
\hline C1s O-C=O & 289.1 & 0.6 \\
\hline O1s C-O & 532.4 & 33.5 \\
\hline O1s -CO- & 531.0 & 2.4 \\
\hline O1s O2*CO & 533.2 & 3.4 \\
\hline N1s NCO & 399.0 & 2.3 \\
\hline N1s NH & 399.9 & 1.6 \\
\hline N1s NH3+ & 401.8 & 0.5 \\
\hline
\end{tabular}

Table S5. Characteristic bonds detected in C1s and N1s peaks of BC-n60ChiD2 sample by XPS method.

\begin{tabular}{|l|l|l|}
\hline Name BC-n60ChiD2 & Binding energy (eV) & Atomic (\%) \\
\hline C1s C-C & 284.6 & 20.1 \\
\hline C1s C-O, C-N & 286.2 & 20.6 \\
\hline C1s O-C-O, NCO & 287.7 & 7.8 \\
\hline
\end{tabular}




\begin{tabular}{|l|l|l|}
\hline C1s O-C=O & 289.0 & 1.2 \\
\hline O1s C-O & 532.4 & 39.3 \\
\hline O1s -CO- & 533.2 & 7.0 \\
\hline O1s O2*CO & 531.0 & 2.1 \\
\hline N1s NCO & 398.8 & 0.6 \\
\hline N1s NH & 399.8 & 1.4 \\
\hline N1s NH3+ & 401.8 & 0.3 \\
\hline
\end{tabular}

Table S6. The position, FWHM and degree of crystallinity of BC, BC-n20ChiD2, BC-n40ChiD2 and BC-n60ChiD2 samples.

\begin{tabular}{|c|c|c|c|}
\hline Sample & $2 \theta\left({ }^{\circ}\right)$ & FWHM & $\begin{array}{l}\text { Degree of } \\
\text { crystallinity }\end{array}$ \\
\hline \multirow[t]{3}{*}{$\mathrm{BC}$} & 14.22 & 1.74 & \multirow[t]{3}{*}{62.6} \\
\hline & 16.74 & 0.92 & \\
\hline & 22.48 & 2.17 & \\
\hline \multirow[t]{3}{*}{ BC-n20ChiD0.2 } & 14.32 & 1.65 & \multirow[t]{3}{*}{75.5} \\
\hline & 16.70 & 0.99 & \\
\hline & 22.46 & 2.73 & \\
\hline \multirow[t]{3}{*}{ BC-n20ChiD2 } & 14.32 & 1.53 & \multirow[t]{3}{*}{70.8} \\
\hline & 16.70 & 0.76 & \\
\hline & 22.46 & 2.94 & \\
\hline \multirow[t]{3}{*}{ BC-n40ChiD0.2 } & 14.32 & 1.65 & \multirow[t]{3}{*}{82.6} \\
\hline & 16.70 & 0.65 & \\
\hline & 22.46 & 2.95 & \\
\hline \multirow[t]{3}{*}{ BC-n40ChiD2 } & 14.32 & 1.65 & \multirow[t]{3}{*}{70.0} \\
\hline & 16.70 & 0.65 & \\
\hline & 22.46 & 2.95 & \\
\hline \multirow[t]{2}{*}{ BC-n60ChiD0.2 } & 14.36 & 1.9 & \multirow[t]{2}{*}{72.0} \\
\hline & 22.62 & 3.75 & \\
\hline \multirow[t]{2}{*}{ BC-n60ChiD2 } & 14.32 & 1.92 & \multirow[t]{2}{*}{74.0} \\
\hline & 22.62 & 3.49 & \\
\hline
\end{tabular}



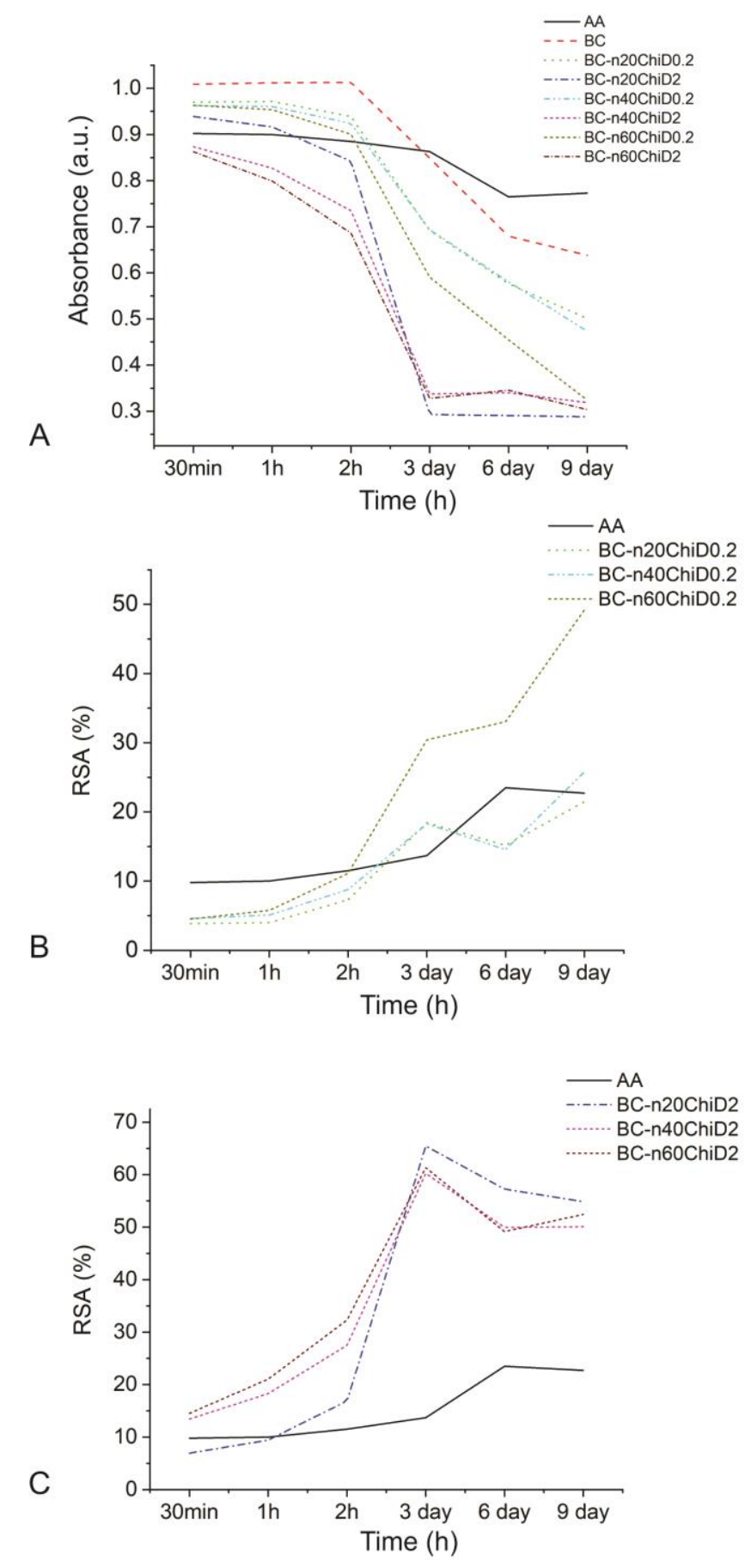

Fig. S4. DPPH• scavenging assay. Absorbance of DPPH• solution with BC, BC-20nChiD0.2, BC-20nChiD2, BC-40nChiD0.2, BC-40nChiD2, BC-60nChiD0.2, BC-60nChiD2 nanocomposites and AA (A); RSA of BC-20nChiD0.2, BC-40nChiD0.2 and BC-60nChiD0.2 nanocomposites and AA (B); RSA of BC-20nChiD2, BC-40nChiD2 and BC-60nChiD2 nanocomposites and $\mathrm{AA}(\mathrm{C})$. 